\title{
Procesos electorales y crisis sanitaria en América Latina. Los desafíos de los organismos electorales
}

\author{
Sofía Vincenzi Guilá* \\ Tasheena Obando Smith**
}

https://doi.org/10.35242/RDE 202222331

Nota del Consejo Editorial

Recepción: 14 de diciembre de 2021.

Revisión, corrección y aprobación: 14 de enero de 2022.

Resumen: Presenta los desafíos que los organismos electorales de América Latina enfrentaron y las medidas que tomaron para que los procesos electorales de la región se celebraran garantizando tanto los derechos políticos de sus ciudadanos como el derecho a la salud. Esto llevó a redefinir las estrategias de comunicación a implementar por parte de los organismos electorales con el fin de transmitir oportunamente las nuevas medidas sanitarias y mitigar el temor de los y las votantes.

Palabras clave: Salud pública / Proceso electoral / Organización electoral / Administración electoral / Votación / Participación ciudadana / Organismos electorales / América Latina.

Abstract: The article presents the challenges and measures that electoral bodies in Latin America faced and took so that electoral processes could take place in the region, guaranteeing the political rights of citizens as well as the right to health, which led to redefining communication strategies to be implemented by the electoral bodies. This was done with the aim of communicating the new sanitary measures in a timely manner and mitigate the fear of voters.

Key Words: Public health / Electoral process / Electoral organization / Electoral administration / Voting / Citizen participation / Electoral bodies / Latin America.

\footnotetext{
* Costarricense, politóloga, correo svincenzi@iidh.ed.cr. Licenciada en Ciencias Políticas por la Universidad de Costa Rica. Funcionaria del Instituto Interamericano de Derechos Humanos (IIDH) y de su Centro de Asesoría y Promoción Electoral (CAPEL) desde 1989, donde actualmente es su coordinadora de operaciones. Ha estado presente en más de 90 procesos electorales como parte de las misiones de observación en América Latina. Ha sido responsable académica de la producción de obras relevantes en doctrina electoral, y coautora del "Diccionario electoral", entre otras obras. Posee una amplia experiencia en la coordinación de la Secretaría Ejecutiva de la Unión Interamericana de Organismos Electorales, la Asociación de Organismos Electorales de Centroamérica y del Caribe y de la Asociación de Organismos Electorales de América del Sur; y la organización de sus actividades. Participó en el Programa de Visitantes a la Unión Europea (EUVP), setiembre-octubre de 2011.

** Costarricense, correo tobando@iidh.ed.cr. Máster en Derechos Fundamentales, por la Universidad Carlos III de Madrid. Licenciada en Relaciones Internacionales con énfasis en Administración y Gerencia de la Cooperación Internacional, por la Universidad Nacional de Costa Rica. Asesora internacional del Centro de Asesoría y Promoción Electoral (CAPEL) del Instituto Interamericano de Derechos Humanos. Experiencia en la elaboración de propuestas de proyectos, informes, en temas vinculados a los derechos políticos; coordinadora técnica de proyectos y publicaciones: Participación Política de las Mujeres, basado en la experiencia centroamericana; trabajo con escuelas e institutos de capacitación de los organismos electorales; experiencia en la organización de cursos y conferencias vinculados a temas electorales; además de haber participado como observadora internacional en más de 30 elecciones de América Latina. Autora en el Diccionario Electoral de IIDH-Capel en su última edición (2016).
} 


\section{DERECHOELECTORAL}

\section{INTRODUCCIÓN}

La aparición de la crisis sanitaria ocasionada por el virus SARS-CoV-2 obligó a replantear la mayoría de las actividades de nuestros países, entre ellas la organización y celebración de los procesos electorales. Estos eventos movilizan una gran cantidad de personas en un solo día y, durante una crisis sanitaria, esto significa un alto riesgo de propagación y contagio de la enfermedad.

Ante una crisis inédita como esta, y con una importante cantidad de procesos electorales calendarizados para los años 2020 y 2021, las autoridades electorales, de manera conjunta con autoridades estatales e instituciones nacionales e internacionales a cargo de la salud, tuvieron que hacer un balance entre la posibilidad de realizar las elecciones, cumplir el orden jurídico y preservar la salud de la ciudadanía. De esta manera, se analizó el dilema entre la protección de los derechos políticos como derechos humanos, pero sin dejar de lado el vínculo que adquieren estos con el derecho a la salud y con el derecho a la vida, al enfrentar una amenaza común, la pandemia y su propagación a partir del ejercicio de los primeros.

Es así como los organismos electorales deben hacer frente a una serie de retos, entre ellos, cómo mitigar el riesgo sanitario, y continuar ejerciendo sus labores y brindando sus servicios a la población; asimismo, llevar a cabo las acciones necesarias para que todas las personas involucradas en los diferentes procesos institucionales, especialmente en la organización de las elecciones, no estuvieran expuestas a contagios. Adicionalmente, a este contexto se sumó el agravante de declaratoria de estado de excepción en la mayoría de los países en los cuales se celebrarían elecciones, en consecuencia, todo el proceso electoral estuvo condicionado a cumplir las medidas sanitarias adoptadas por los Gobiernos, lo que cambió las condiciones en las cuales se organizaban las elecciones; y la participación ciudadana indefectiblemente debió variar en medio de estados de emergencia.

\section{LOS DESAFÍOS DE LOS ORGANISMOS ELECTORALES}

Los organismos electorales enfrentaron desafíos considerables debido a la pandemia, algunos de origen político, relacionados con las decisiones en 


\section{DERECHO ELECTORAL}

torno a la posible postergación de las elecciones y otros vinculados con la nueva atribución que adquieren con la necesidad de implementar medidas sanitarias en el desarrollo de sus funciones. (Romero, 2020, p.34). Por eso, estas entidades se vieron en la posición de seguir adelante con el ciclo electoral planificado, con ajustes ineludibles al calendario electoral y con la aparición de nuevos actores fundamentales en el momento de toma de decisiones, por ejemplo, las instituciones encargadas de la salud, Gobiernos y demás autoridades que velaban por el respeto a las nuevas regulaciones sanitarias.

En el transcurso del año 2020 y principios del 2021, hubo cambios significativos en las fechas programadas para la celebración de las elecciones a nivel mundial, muchas modificadas dentro del marco constitucional permitido, mientras otras por medio de medidas transitorias extraordinarias. Para efectos de este artículo es importante destacar que en América Latina la mayoría de los países regula lo relativo a la materia electoral desde la Constitución misma, incluyendo en algunos casos las fechas específicas para la celebración de los comicios o traspaso de autoridades electas. Asimismo, cualquier cambio realizado en el calendario electoral debió contar con un verdadero consenso o una mayoría muy sólida entre las fuerzas políticas, sobre todo en aquellos casos en los que se debió promulgar una ley que permitiera aprobar las modificaciones, interpretaciones, normas temporales o respaldar las determinaciones y recomendaciones del organismo electoral.

Lo cierto es que en la región se postergó un número importante de elecciones, con el fin de proteger la salud de las y los ciudadanos (y de la población en general); primordialmente durante el 2020, ello con el objetivo de conocer un poco más acerca del virus, tomar las medidas necesarias para el trabajo de los organismos electorales y poder redactar e implementar los protocolos sanitarios necesarios para seguir trabajando y realizar las elecciones. A continuación, se citan algunos ejemplos de países en los cuales las fechas de las elecciones cambiaron durante ese periodo.

República Dominicana: La Junta Central Electoral (JCE) realizó el cambio de la fecha, previa consulta con los partidos políticos y en un contexto de toque de queda. La JCE determinó postergar la elección por un asunto de fuerza mayor, mediante la resolución n. ${ }^{\circ}$. 42/2020 (acto administrativo). 


\section{DERECHO ELECTORAL}

Uruguay: La Ley 19.875 fue publicada el 8 de abril de 2020, y facultó a la Corte Electoral (CE) y a los mismos legisladores a tomar las medidas necesarias para postergar las actividades del proceso electoral subnacional. El 22 de abril del mismo año, la CE anunció el 27 de septiembre como la nueva fecha para realizar las elecciones.

Bolivia: El Tribunal Supremo Electoral (TSE) cuando se decreta la pandemia suspende el calendario electoral y convoca a los candidatos a una mesa de diálogo con el fin de analizar alternativas para reanudar el proceso electoral. TSE definió el 18 de octubre como la nueva fecha para las elecciones con base en criterios técnicos y sanitarios, y lo planteó a la Asamblea Legislativa por medio de un proyecto de ley; posteriormente, la Asamblea Legislativa aprobó dicha reprogramación.

Chile: La nueva fecha del Plebiscito Nacional la propuso el Poder Ejecutivo. El Servicio Electoral de Chile (SERVEL) dio las consideraciones técnicas. Mediante Ley número 21.221 del Congreso Nacional se aprueba la reforma constitucional que establece el nuevo itinerario electoral para el plebiscito nacional y otros eventos electorales.

Brasil: El Tribunal Superior Electoral (TSE), en consulta con expertos en salud y debido a la crisis sanitaria, decidió que el cambio de fecha de las Elecciones Municipales sería necesario para garantizar la seguridad de las personas. El Congreso hizo lo mismo, y el acuerdo fue cambiar la fecha para noviembre de 2020. Dicha reprogramación se aprobó por medio de una ley en el Congreso Nacional.

Paraguay: En medio de la declaratoria de estado de emergencia, y después de una comparecencia en el Congreso, el 23 de marzo de 2020 el Tribunal Superior de Justicia Electoral (TSJE) dispuso postergar por primera vez las fechas de las elecciones primarias y la jornada de votación municipal -al 2 de agosto y 29 de noviembre de 2020 respectivamente-. Finalmente, dichos procesos electorales se realizaron el 20 de junio y el 10 de octubre de 2021.

México: Mediante la "facultad de atracción" el Instituto Nacional Electoral (INE) puede tomar decisiones locales. Para las elecciones en Coahuila e Hidalgo, el INE pospuso la fecha del 7 de junio al 18 de octubre de 2020.

Seguidamente, en la tabla 1 , se identifica la totalidad de procesos electorales en América Latina para el 2020, calendarizados previo a la declaratoria de pandemia, y la fecha en la que tuvieron lugar después de 


\section{DERECHO ELECTORAL}

su posposición. Como se observa, hubo casos en los que el cambio de fecha implicó la realización de elecciones el siguiente semestre y, algunos pocos, como el caso de Paraguay, un año después.

Tabla 1

Cambio en el calendario electoral 2020

\begin{tabular}{|c|c|c|c|}
\hline Fecha original & Fecha nueva & Tipo de elección & País \\
\hline 26 de abril & 25 de octubre & Plebiscito & Chile \\
\hline 3 de mayo & 18 de octubre & Elecciones presidenciales & Bolivia \\
\hline 10 de mayo & 27 de setiembre & $\begin{array}{l}\text { Elecciones departamentales y } \\
\text { municipales }\end{array}$ & Uruguay \\
\hline 17 de mayo & 5 de julio & Elecciones generales & $\begin{array}{l}\text { República } \\
\text { Dominicana }\end{array}$ \\
\hline 7 de junio & 18 de octubre & Elecciones en Coahuila e Hidalgo & México \\
\hline 14 de junio & 29 de noviembre & Segunda vuelta presidencial & Bolivia \\
\hline 12 de julio & 20 de junio, 2021 & Elecciones internas de partidos políticos & Paraguay \\
\hline 4 de octubre & 15 de noviembre & Elecciones municipales & Brasil \\
\hline 25 de octubre & 11 de abril, 2021 & Elecciones municipales & Chile \\
\hline 25 de octubre & 29 de noviembre & Segunda vuelta municipal & Brasil \\
\hline 4 de octubre & 15 de noviembre & Elecciones municipales & Brasil \\
\hline 25 de octubre & 11 de abril, 2021 & Elecciones municipales & Chile \\
\hline 25 de octubre & 29 de noviembre & Segunda vuelta municipal & Brasil \\
\hline \multirow[t]{2}{*}{8 de noviembre } & 10 de octubre, 2021 & Elecciones municipales & Paraguay \\
\hline & 06 de diciembre & Elecciones parlamentarias & Venezuela \\
\hline
\end{tabular}

Nota: Elaborado con base en los datos de IIDH/CAPEL.

La tabla 2 hace referencia a la calendarización de elecciones programadas y realizadas durante el año 2021, todavía en un contexto de crisis sanitaria y tomando las medidas necesarias para garantizar la salud de todas las personas involucradas en la organización del proceso, actores políticos, funcionarias y funcionarios electorales y los ciudadanos y ciudadanas. 


\section{DERECHO EIECTORAL}

Tabla 2

Calendario electoral 2021

Fecha
Tipo de elección

Elecciones presidenciales, asambleístas y parlamentarios andinos

Elecciones de diputados, diputadas al Parlamento Centroamericano, Asamblea Legislativa e integrantes de concejos municipales

Elecciones subnacionales

Elecciones primarias

Segunda vuelta elecciones subnacionales

Elecciones generales

Elecciones de gobernadores regionales, alcaldes y concejales y eventuales convencionales constituyentes

Segunda vuelta elecciones presidenciales

Elección de diputados federales y autoridades de las 32 entidades del país

Elecciones internas simultáneas

Elecciones primarias

Elecciones PASO

Elecciones municipales

Elecciones generales

Elecciones legislativas generales

Elecciones presidenciales, parlamentarias y de consejeros regionales

Elecciones generales

19 de diciembre Segunda vuelta presidencial

País

Ecuador

El Salvador

Bolivia

Honduras

Bolivia

Perú

Chile

Perú

México

Paraguay

Chile

Argentina

Paraguay

Nicaragua

Argentina

Chile

Honduras

Chile

Nota: Elaborado con base en los datos de IIDH/CAPEL.

Es importante señalar que los comicios enumerados en la tabla 2 se realizaron y que los organismos electorales han logrado implementar de manera gradual mecanismos para hacer frente a la pandemia y los retos que esto trae consigo.

Otro desafío consistió en mantener la confianza ciudadana en que la autoridad electoral iba a resguardar la salud de todas las personas 


\section{DERECHO EIECTORAL}

involucradas en el proceso, durante sus distintas etapas en el contexto de crisis sanitaria, generar la credibilidad en los comicios y, a su vez, ofrecer las condiciones necesarias para su legitimidad. En este sentido, se requirió la redefinición de la estrategia de comunicación que se implementaría con el fin de transmitir oportunamente las nuevas medidas sanitarias y mitigar el temor de los y las votantes, buscando asegurar que confiaran en los protocolos diseñados y en la eficacia de las medidas tomadas para resguardar la integridad del sufragio sin poner en riesgo su salud.

Ante este panorama, la estrategia de comunicación debía combatir la desinformación, prevenir el temor de los electores a contagiarse, pero principalmente propiciar un ambiente seguro en el que la persona que vota pueda participar (Tullio, 2020, p. 15). La experiencia acumulada en la organización de procesos electorales en América Latina coincide en que las estrategias de información y la comunicación a tiempo son exitosas en el tanto se hagan con antelación y tengan buena difusión. En este contexto, no puede ser diferente cuando se refiere a las medidas y protocolos sanitarios aprobados, ya que proveen mayor tranquilidad a los y las votantes.

Un tercer desafío que enfrentaron los organismos electorales como consecuencia de la pandemia fue continuar con sus funciones cotidianas y sobre todo con la organización de los procesos electorales programados. Como fue el caso de aquellos que tuvieron lugar el segundo semestre de 2020, donde se pudo identificar nuevas condiciones para la organización y la participación ciudadana, entre ellas:

- Asegurar la salud de las personas funcionarias electorales.

- Identificar las etapas del proceso electoral que se podían ver más afectadas.

- Asegurar los niveles de participación que garantizaran la legitimidad de las autoridades electas (evitar el ausentismo):

- Asegurar un entorno de votación seguro (minimizando los riesgos de contagio).

- Reclutar personal adicional para trabajar en los recintos electorales (para velar por el cumplimiento de medidas sanitarias).

- Asegurar la equidad para los diferentes actores en contienda. 


\section{DERECHO ELECTORAL}

- Conocer los requisitos impuestos en los países donde se implemente el voto en el extranjero.

- Modificar los materiales electorales y adicionar un kit con materiales de bioseguridad.

- Aumentar el uso de tecnología tanto en la prestación de servicios como en el desarrollo de las funciones (trámites, habilitación de trabajo remoto, sesiones de las autoridades y funcionarios, capacitación, entre otros).

De manera general se puede afirmar que las entidades electorales demostraron su carácter de resiliencia y rápidamente dieron respuesta a todos los desafíos que se presentaron, y para poder hacerlo, fueron fundamentales los espacios de cooperación horizontal que se propiciaron en el marco de las asociaciones regionales de organismos electorales. La experiencia se fue construyendo de manera paulatina en los países de la región, la cual inició con la elección presidencial y congresual en República Dominicana que tuvo lugar el 5 de julio de 2020, la primera en el continente en un contexto de crisis sanitaria.

En este sentido, los eventos virtuales de intercambio de experiencias entre organismos electorales que ya habían celebrado elecciones y los que estaban apenas organizándolas se convirtieron en una herramienta de uso común, principalmente en temas como la elaboración de protocolos sanitarios, de estrategias de información y combate a la desinformación, así como eventos enfocados en compartir experiencias en la organización de elecciones en pandemia, tomando en cuenta las nuevas prioridades identificadas. Un ejemplo de esto fueron las diferentes reuniones virtuales que se gestaron a nivel internacional, entre las que destacan las realizadas en el marco de la Unión Interamericana de Organismos Electorales y que han sido coordinadas desde el Centro de Asesoría y Promoción Electoral (IIDH/CAPEL).

\section{PROTOCOLOS SANITARIOS Y ALGUNAS MEDIDAS IMPORTANTES}

La incertidumbre que rodeó la pandemia generó la necesidad de contar con instrumentos prácticos que regularan y promovieran la celebración de los comicios de manera segura, y evitar, en la medida de las posibilidades, el aumento de contagios debido a las aglomeraciones no solo el día de las elecciones, sino también los días previos y posteriores. Para esto, los organismos electorales optaron por la elaboración de protocolos que aplicaran medidas sanitarias en todas las etapas del proceso, de manera 


\section{DERECHO EIECTORAL}

tal que se armonizara la protección ciudadana con el ejercicio de los derechos políticos.

En general, los protocolos sanitarios buscan la reducción de riesgos de contagio mediante la definición de medidas de higiene, materiales de bioseguridad y capacitación a los actores que intervienen en el proceso electoral. El objetivo principal de los protocolos sanitarios ha sido asegurar que los y las ciudadanas puedan participar de manera activa a lo largo de todo el proceso electoral, tanto en su organización y en las campañas electorales como el día de la jornada electoral y en la etapa posterior, con la seguridad de que su salud estará protegida.

Como ejemplo se señalan algunas de las medidas adoptadas en las últimas elecciones de Bolivia, Chile y Perú.

\section{Bolivia:}

- Distribuir a los electores para emitir el voto de acuerdo con el último número de su documento de identidad.

- Seleccionar a los jurados electorales entre personas de 18 y 50 años.

- Promover que cada elector llevara su lapicero.

- Extender el número de mesas de votación.

- Aumentar la cantidad de centros de votación.

\section{Chile:}

- Promover que los electores llevaran su propio lápiz.

- Nombrar facilitadores para implementar el protocolo sanitario.

- Excusar de que fueran a votar a las personas con alguna discapacidad o mayores de 60 años.

- Aumentar el número de centros de votación.

- Prohibir que personas enfermas de COVID-19 votaran.

- Ampliar a dos días la jornada de votación.

\section{Perú:}

- Eliminar el cobro de multas por no votar a las personas mayores de 60 años o con alguna discapacidad.

- Excusar de ser miembros de mesa a personas con alguna condición de vulnerabilidad.

- Seleccionar centros de votación con espacios abiertos. 


\section{DERECHO ELECTORAL}

- Instar a los electores para que elijan su centro de votación dentro de su jurisdicción.

En la mayoría de los países que tuvieron elecciones entre el segundo semestre del 2020 y a lo largo del 2021, la implementación oportuna de las medidas sanitarias y su correcta difusión a toda la población tuvo resultados positivos. En algunos, no hubo un aumento de casos de COVID-19 posterior a las elecciones, mientras que en otros disminuyeron, y en aquellos donde hubo un aumento fue porque la elección se realizó durante un pico de contagios y ya se sabía de antemano que eso iba a suceder.

Por otra parte, se debe reconocer que no en todos los casos las medidas sanitarias inicialmente definidas funcionaron de manera exitosa, sin embargo, hubo países en los que se celebró más de un evento electoral y se pudieron rectificar las medidas, con el objetivo de robustecerlas y proteger a las personas votantes. Algunos de estos casos se resumen a continuación.

Ecuador (Elecciones generales y segunda vuelta presidencial):

- El ingreso a los centros de votación inicialmente fue regulado durante la primera vuelta; esto ocasionó grandes aglomeraciones en las afueras. Para la segunda ronda presidencial, se removió la restricción y las filas se regularon dentro de los centros, lo que hizo más ágil la votación.

- Entre la elección general y la segunda vuelta presidencial hubo un incremento del personal sanitario que asistía en los recintos.

Chile (Plebiscito Nacional y elecciones de gobernadores regionales, alcaldes, concejales y eventuales convencionales constituyentes):

- Las elecciones pasaron de realizarse en un día a llevarse a cabo en dos días, como medida para evitar aglomeraciones.

- Se reforzó el kit sanitario en cada evento electoral.

- Entre cada elección, se realizaron mesas de trabajo con sociedad civil para validar protocolos a utilizar.

Perú (elecciones generales y segunda vuelta presidencial):

- Se incorporó el uso de la doble mascarilla para ingresar a los locales de votación, en la medida que la obligación existió en el último decreto 


\section{supremo emitido por el Gobierno como política en salud y prevención del COVID.}

- Como estrategia, se modificó la disposición de horario reservado para adultos mayores, mujeres embarazadas, personas con discapacidad o en riesgo en caso de contagio.

Seguidamente, en la figura 1 , se señalan algunas de las consideraciones que han tenido como punto de partida los organismos electorales en el momento de redactar sus protocolos sanitarios. Según cada país, estas han debido tomar en cuenta el marco legal que rige cada elección.

\begin{tabular}{|c|c|c|c|}
\hline $\begin{array}{l}\text { Campañas electorales } \\
\text { Uso de redes sociales en campaña } \\
\text { electoral (definir reglamento). } \\
\text { Regular manifestaciones y } \\
\text { reuniones. }\end{array}$ & \multicolumn{2}{|c|}{$\begin{array}{l}\text { Capacitación ciudadana } \\
\text { Diseño de estrategia de } \\
\text { comunicación digital, radio y tv. } \\
\text { Campaña de educación sobre } \\
\text { nuevas medidas de participación } \\
\text { ciudadana y sobre protocolo } \\
\text { sanitario. }\end{array}$} & $\begin{array}{l}\text { Personal a cargo de procesos en las } \\
\text { elecciones } \\
\text { Pruebas de COVID-19 al personal electoral. } \\
\text { Brindar capacitaciones virtuales a } \\
\text { miembros de mesa y otros funcionarios. } \\
\text { Designar personal auxiliar que supervise el } \\
\text { cumplimiento de medidas de higiene en } \\
\text { centros de votación con la ayuda de la } \\
\text { Fuerza Pública. }\end{array}$ \\
\hline $\begin{array}{l}\text { Materiales adicionales en el kit } \\
\text { electoral (kit electoral } \\
\text { adicional) } \\
\text { Alcohol en gel, mascarillas, } \\
\text { guantes, caretas, toallas de } \\
\text { desinfección. } \\
\text { Cartilla de capacitación sobre uso } \\
\text { de protocolo sanitario. }\end{array}$ & \multicolumn{2}{|c|}{$\begin{array}{l}\text { Preparación y distribución de } \\
\text { material electoral } \\
\text { Distanciamiento físico entre el } \\
\text { personal a cargo de la } \\
\text { preparación de materiales } \\
\text { electorales. } \\
\text { Brindar insumos de higiene } \\
\text { necesarios para la protección del } \\
\text { personal. } \\
\text { Designar personal encargado de } \\
\text { desinfectar el exterior del kit } \\
\text { electoral antes de ser entregado } \\
\text { en los centros de votación. }\end{array}$} & $\begin{array}{l}\text { Material sanitario para el centro de } \\
\text { votación } \\
\text { Alcohol en gel para ubicar en la entrada del } \\
\text { centro de votación y adicional para reponer } \\
\text { en las mesas que lo requieran, mascarillas } \\
\text { para brindar a los electores antes de } \\
\text { ingresar al centro de votación (estas } \\
\text { deberán entregarse en paquete individual a } \\
\text { cada elector) y jabón líquido para } \\
\text { estaciones de lavado de mano. }\end{array}$ \\
\hline \multicolumn{2}{|c|}{$\begin{array}{l}\text { Locales de votación } \\
\text { Desinfectar locales de votación antes del inicio de las } \\
\text { elecciones. } \\
\text { Estaciones de lavado de manos y desinfección a la } \\
\text { entrada y dentro de los locales de votación. } \\
\text { Información sobre las medidas que deben seguir los } \\
\text { electores para ingresar al centro. } \\
\text { Disminuir el número de votantes por junta receptora } \\
\text { de votos (incrementar los locales de votación). } \\
\text { Utilizar termómetros en la entrada del centro de } \\
\text { votación para determinar la temperatura de los } \\
\text { electores. } \\
\text { Desinfectar la mampara y la mesa antes de su uso } \\
\text { por cada votante. En caso de que haya voto } \\
\text { electrónico, desinfectar la máquina utilizada. } \\
\text { Evitar aglomeraciones fuera de los centros de } \\
\text { votación. La Fuerza Pública debe tener un rol en esta } \\
\text { medida. } \\
\text { Reorganizar el diseño de la mesa electoral para } \\
\text { minimizar el contacto entre sus miembros y entre } \\
\text { estos y los votantes, manteniendo } 2 \text { metros de } \\
\text { distancia. } \\
\text { Señalar el espacio de cada votante para hacer la fila } \\
\text { de ingreso al centro de votación y fuera de su mesa } \\
\text { de votación. }\end{array}$} & \multicolumn{2}{|c|}{$\begin{array}{l}\text { Escrutinio y transmisión de resultados } \\
\text { Previo al escrutinio, desinfectar todo el centro de votación y } \\
\text { los materiales y equipo utilizado para el proceso. } \\
\text { Brindar un instructivo para el escrutinio con medidas de } \\
\text { distanciamiento e higiene. } \\
\text { Considerar la posibilidad de llenar el acta en formato } \\
\text { digital. } \\
\text { Definir condiciones de delegados de partidos políticos con el } \\
\text { fin de guardar la distancia durante el escrutinio. } \\
\text { Considerar nuevas herramientas informáticas para agilizar } \\
\text { la transmisión de resultados. }\end{array}$} \\
\hline
\end{tabular}




\section{DERECHO ELECTORAL}

Figura 1.Consideraciones para el establecimiento de un protocolo sanitario. Elaborado con base en el Fascículo 1. Serie: Elecciones en tiempos de pandemia. Consideraciones para el establecimiento de un protocolo para la celebración de elecciones en el contexto de una crisis sanitaria. (IIDH/CAPEL, 2020a).

\section{Cambios ADMINISTRATIVOS EN ORGANISMOS Electorales}

La pandemia ha representado la oportunidad para que los organismos electorales innoven en el ejercicio de sus funciones; y la adopción de nuevas tecnologías ha facilitado la implementación de iniciativas que, sin poner en riesgo la integridad de los procesos, permitan continuar con sus obligaciones. Algunas de las decisiones tomadas para continuar funcionando en el marco de la crisis sanitaria y en medio de restricciones y medidas sanitarias se señalan a continuación:

- Protección de funcionarios y ciudadanos que deben acudir a las instalaciones: puesta en práctica de protocolos de bioseguridad.

- Trabajo remoto de los funcionarios: reestructurar sistemas de información y diseñar aplicativos que permitan al personal el trabajo remoto.

- Sesiones del pleno de los organismos electorales vía virtual.

- Extensión de validez de los documentos de identidad.

- Consultas de trámites civiles por teléfono (número único).

- Atención mediante citas (solicitadas en página web).

- Continuación de programas de capacitación virtuales.

- Certificaciones digitales (nacimiento, defunción, matrimonio).

- Notificaciones digitales (organismos jurisdiccionales).

- Aplicaciones para consultar lugar de votación, miembros de las JRV, padrón electoral, registro de votantes en el extranjero.

En un balance general y a nivel de la UNIORE, se ha considerado que todas estas medidas han contribuido a que los organismos electorales no dejaran de dar servicios a los ciudadanos y a las ciudadanas, inclusive algunas continúan vigentes. De la misma manera, las experiencias han podido ser compartidas entre los miembros de UNIORE, con el fin de brindar cooperación horizontal que a corto plazo pudiera contribuir en la organización de las elecciones que todavía estaban por realizarse. Para esto, el reconocimiento de procedimientos innovadores mediante la 


\section{DERECHO ELECTORAL}

utilización de herramientas tecnológicas ha permitido que los organismos electorales puedan a partir de estas experiencias adaptar sus procedimientos y facilitar el ejercicio de los derechos políticos de la población.

\section{CONCLUSIONES}

Muchos países, y sus instituciones, se enfrentaron al dilema de celebrar o postergar los procesos electorales programados en los meses posteriores a la declaratoria del COVID-19 como pandemia. Decisión difícil porque, como ya se dijo, se tuvo que hacer un balance entre el derecho a la salud y los derechos políticos.

En un primer momento, y por la gravedad de la situación, en América Latina se optó por la postergación de las elecciones y consultas programadas durante el año 2020 y algunas en el año 2021, pero estas no se podían posponer por siempre y se debió tomar acciones para realizar las elecciones preservando la salud de las personas. Es así como las autoridades estatales y los organismos electorales se esforzaron para garantizar la integridad de los procesos y sus resultados, ofreciendo las mejores condiciones sanitarias para los participantes. De igual forma, los protocolos sanitarios para implementar a lo largo de todo el proceso electoral y para el trabajo cotidiano de los organismos electorales se volvieron indefectibles, se precisaron los materiales de bioseguridad necesarios, se evaluaron las estrategias de información y comunicación, entre otras acciones necesarias, y que se definieron en el marco de la Constitución, las leyes y los reglamentos de cada país.

La implementación de los protocolos sanitarios por parte de los organismos electorales se ha considerado exitosa y las elecciones no tuvieron una incidencia en la evolución de la pandemia. Para lograrlo, fue necesario que los organismos electorales y las autoridades estatales tomara las medidas adecuadas, pero también fue importante que la ciudadanía, de manera responsable, respetara las disposiciones establecidas en estos protocolos.

También se rescata la importancia de la redefinición de las estrategias de comunicación e información de parte de los organismos electorales. Estas resultaron indispensables para compartir las nuevas medidas adoptadas para facilitar un voto seguro, educar al elector y generar tranquilidad y confianza. 


\section{DERECHO ELECTORAL}

En los próximos años, los procesos electorales no pueden obviar los temas de higiene y salud pública. Los protocolos sanitarios llegaron para quedarse en la organización de cualquier proceso y a lo largo de todas sus etapas.

En este momento existen referentes, experiencia acumulada y conocimiento relevante a fin de incorporar en la planificación de los procesos electorales los mecanismos necesarios para hacer frente a los problemas de contagios en el proceso electoral. De esto es ejemplo el trabajo desarrollado en el marco de UNIORE. En ese sentido, los organismos electorales han puesto en evidencia su capacidad de reinventarse dando soluciones oportunas, adecuadas y sostenibles basadas en su experiencia o en la de sus iguales.

\section{Referencias bibliográficas}

Conversatorio virtual "Organizando elecciones durante la pandemia del Covid-19: el caso de América Latina" (11 de mayo de 2020). Organizado por el Instituto Nacional Electoral de México y el Centro de Asesoría y Promoción Electoral del Instituto Interamericano de Derechos Humanos. Recuperado de https://www.youtube.com/watch?v=_Mu91pLp1dQ

Instituto Interamericano de Derechos Humanos (2020). Protocolos sanitarios de países de América Latina que han tenido elecciones en los años 2020 y 2021 y cápsulas informativas elaboradas por organismos electorales en las cuales explican las principales medidas administrativas. Recuperado de https://www.iidh.ed.cr/capel/elecciones-y-covid19

Instituto Interamericano de Derechos Humanos. Centro de Asesoría y Promoción Electoral. (2020a). Consideraciones para el establecimiento de un protocolo para celebración de elecciones en el contexto de una crisis sanitaria. Fascículo 1, Serie de fascículos América Latina: Elecciones en tiempos de pandemia. San José, C.R.: IIDH.

Instituto Interamericano de Derechos Humanos. Centro de Asesoría y Promoción Electoral (2020b). El Covid-19 y las elecciones en América Latina. Fascículo 3, Serie de fascículos América Latina: Elecciones en tiempos de pandemia. San José, C.R.: IIDH.

Romero, S. (2020). Experiencia de organismos electorales de América Latina en la celebración de procesos electorales en pandemia. Fascículo 9, Serie de fascículos América Latina: Elecciones en tiempos de pandemia, San José, C.R.: IIDH. 


\section{DERECHO ELECTORAL}

Tullio, A. (2020). Lecciones aprendidas en materia de organización de elecciones en contexto de pandemia y post-pandemia. Fascículo 4, Serie de fascículos América Latina: Elecciones en tiempos de pandemia. San José, C.R.: IIDH.

\section{Informes de Misiones de Observación de la UNIORE}

Informes de las Misiones de Observación de la Unión Interamericana de Organismos Electorales para los siguientes procesos electorales:

- Informe Elecciones Presidenciales República Dominicana, julio, 2020

- Informe Elecciones Presidenciales Bolivia, octubre, 2020

- Informe Elecciones de Diputados y Municipales El Salvador, febrero, 2021

- Informe Elecciones Generales Ecuador, febrero-abril, 2021

- Informe Elecciones Sub Nacionales Bolivia, marzo, 2021

- Informe Elecciones Primarias Honduras, marzo, 2021

- Informe Elecciones Generales Perú, abril, 2021

- Informe Segunda vuelta Elecciones Presidenciales Perú, junio, 2021

- Informe lección de diputados federales y autoridades de las 32 entidades del país México, junio, 2021

- Informe Elecciones Internas Simultáneas Paraguay, junio, 2021 